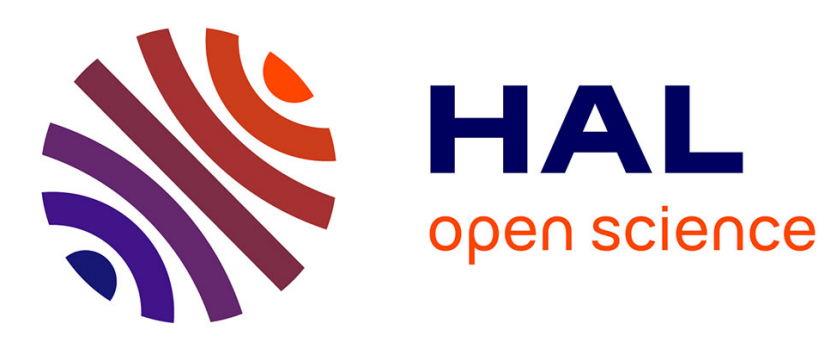

\title{
A simulation tool for brassiness studies
}

\author{
Joël Gilbert, Ludovic Menguy, Murray Campbell
}

\section{To cite this version:}

Joël Gilbert, Ludovic Menguy, Murray Campbell. A simulation tool for brassiness studies. Journal of the Acoustical Society of America, 2008, 123 (4), pp.1854-1857. hal-00207999

\section{HAL Id: hal-00207999 \\ https://hal.science/hal-00207999}

Submitted on 18 Jan 2008

HAL is a multi-disciplinary open access archive for the deposit and dissemination of scientific research documents, whether they are published or not. The documents may come from teaching and research institutions in France or abroad, or from public or private research centers.
L'archive ouverte pluridisciplinaire HAL, est destinée au dépôt et à la diffusion de documents scientifiques de niveau recherche, publiés ou non, émanant des établissements d'enseignement et de recherche français ou étrangers, des laboratoires publics ou privés. 


\title{
A simulation tool for brassiness studies
}

\author{
Joël Gilberta) and Ludovic Menguy \\ Laboratoire d'Acoustique de l'Université du Maine, \\ UMR CNRS 6613, \\ Le Mans, \\ France \\ Murray Campbell \\ University of Edinburgh, \\ Edinburgh EH9 3JZ, \\ $U K$ \\ (Dated: January 17, 2008)
}




\begin{abstract}
A frequency-domain numerical model of brass instrument sound production is proposed as a tool to predict their brassiness, defined as the rate of spectral enrichment with increasing dynamic level. It is based on generalized Burger's equations dedicated to weakly nonlinear wave propagation in non uniform ducts, and is an extension of previous work by Menguy and Gilbert (2000), initially limited to short cylindrical tubes. The relevance of the present tool is evaluated by carrying out simulations over distances longer than typical shock formation distances, and by doing preliminary simulations of periodic regimes in a typical brass trombone bore geometry.
\end{abstract}

PACS numbers: 43.75.Fg and 43.25.Gf 


\section{INTRODUCTION}

At high dynamic levels brass instruments generate sounds having strong high frequency components; these sounds are called "brassy" or "cuivrés". They are due to the essential nonlinearity of the wave propagation in the pipe (Hirschberg et al, 1996). The brassiness of the instruments defined as the rate of spectral enrichment with increasing dynamic level, can be very different due to the variety of their bore geometry. For example a conical bore implies a faster decay of the wave than a cylindrical bore, which reduces the nonlinear wave steepening. Recently Gilbert (2006), Myers et al (2007), and Gilbert et al (2007) have suggested classifying brass instruments from the brassiness point of view. A numerical model would be a useful tool to investigate the brassiness behavior of brass instruments characterized by their bore geometry. Numerical models have been developed in the time domain (Msallam et al, 2000; Vergez and Rodet, 2000), or in the frequency domain (Thompson and Strong, 2001) by making some restrictive assumptions like modeling the wave steepening only on a part of the bore or ignoring backward nonlinear wave propagation.

In this letter we present a simulation tool in the frequency domain extending the work of Menguy and Gilbert (2000), which was limited to short cylindrical tubes. Brass instruments are characterized by non uniform ducts which can be significantly longer than typical shock formation distances. It is shown (section II) that the tool is relevant first, by exploring the weakly nonlinear wave propagation at long distances, and second, by doing preliminary simulations of periodic regimes in a typical brass trombone bore geometry (section III).

\section{WEAKLY NONLINEAR ACOUSTIC SIMULATIONS IN DUCTS}

\section{A. Theoretical background}

The study of weakly nonlinear propagation in a dissipative viscothermal homogeneous fluid assuming a one-dimensional flow in a nonuniform duct leads to first-order nonlinear

a)Electronic address: Joel.Gilbert@univ-lemans.fr 
differential twin equations using the dimensionless variables $\sigma$ (a slow geometric scale equal to $(\gamma+1) / 2 . M . x$ where $\gamma$ is the specific heat ratio, $M$ a Mach number, $x$ a dimensionless geometric scale), $\theta_{+}$and $\theta_{-}$(dimensionless delayed time scales), $q_{+}$and $q_{-}$(forward and backward variables). They are the "generalized Burgers equation" for the forward-traveling wave (equation 1), and for the backward-traveling wave (equation 2):

$$
\begin{aligned}
& \frac{\partial q_{+}}{\partial \sigma}-q_{+} \frac{\partial q_{+}}{\partial \theta_{+}}+q_{+} \frac{\partial \ln (D(\sigma) / D(0))}{\partial \sigma}=+\frac{1}{\Gamma} \frac{\partial^{2} q_{+}}{\partial \theta_{+}{ }^{2}}-\frac{T}{\epsilon} \frac{\partial^{1 / 2} q_{+}}{\partial \theta_{+}{ }^{1 / 2}}, \\
& \frac{\partial q_{-}}{\partial \sigma}+q_{-} \frac{\partial q_{-}}{\partial \theta_{-}}-q_{-} \frac{\partial \ln (D(\sigma) / D(0))}{\partial \sigma}=-\frac{1}{\Gamma} \frac{\partial^{2} q_{-}}{\partial \theta_{-}{ }^{2}}+\frac{T}{\epsilon} \frac{\partial^{1 / 2} q_{-}}{\partial \theta_{-}{ }^{1 / 2}},
\end{aligned}
$$

where $\Gamma$, the Gold'berg number, is a measure of the importance of effects of volume dissipation compared to those of nonlinearity. A plane wave that propagates in a duct with rigid walls experiences dispersion and attenuation as a result of the viscothermal boundary layer along the walls. As a consequence, the right hand side of equations 1 and 2 contains a second term controlled by the dimensionless number $T / \varepsilon$ (Menguy and Gilbert, 2000) which is a measure of the strength of nonlinearity relative to that of wall dissipation. If the volume viscothermal effects - controlled by $\Gamma$ - are frequency squared dependent, the classical dependence of the viscothermal boundary-layer losses - controlled by $T / \varepsilon$ - is on the square root of the frequency. The left hand of equations 1 and 2 exhibit a term which is function of the interior diameter $D(x)$ of the nonuniform duct, characterizing its slowly varying cross section. This term is linear in $q$. It is assumed that the duct diameter D is small enough ( $k D<1, k$ being the wave number), and that the area varies sufficiently slowly on the scale of a wavelength, $(1 / k D) \cdot(d D / d x)<<1$, to justify a one-dimensional propagation model.

The two waves are assumed to propagate in opposite directions, independently in the linear limit. They are non integrable, and there is almost no chance of general analytical progress (Hamilton and Blackstock, 1998). That is why numerical methods such as the one described in the following section should be used. 


\section{B. Simulations in uniform and nonuniform ducts}

Generalized Burger's equations 1 and 2 have no known analytical solutions. Numerical solutions are performed in the frequency domain, the method described in Menguy and Gilbert (2000) is summarized hereafter.

The following simple wave $q_{+}(\sigma, \theta)$ is considered:

$$
q_{+}(\sigma, \theta)=\sum_{n=1}^{\infty}\left[a_{n}(\sigma) \sin n \theta+b_{n}(\sigma) \cos n \theta\right] .
$$

Equalizing each term of the Fourier series coming from equation 1 leads, for each harmonic component $n$, to:

$$
\left\{\begin{array}{c}
\frac{\partial a_{n}}{\partial \sigma}=n\left(\sum_{p=1}^{n-1}\left(\frac{a_{p} a_{n-p}}{2}-\frac{b_{p} b_{n-p}}{2}\right)\right. \\
\left.-\sum_{p=n+1}^{+\infty}\left(a_{p-n} a_{p}+b_{p} b_{p-n}\right)\right)+\frac{1}{\Gamma} n^{2} a_{n} \\
-a_{n}\left(\frac{\partial \ln (D(\sigma) / D(0))}{\partial \sigma}\right)-\frac{T}{\epsilon} \frac{D(0)}{D(\sigma)} \sqrt{\frac{n}{2}}\left(a_{n}-b_{n}\right) \\
\frac{\partial b_{n}}{\partial \sigma}=n\left(\sum_{p=1}^{n-1}\left(\frac{a_{p} b_{n-p}}{2}+\frac{b_{p} a_{n-p}}{2}\right)\right. \\
\left.+\sum_{p=n+1}^{+\infty}\left(b_{p-n} a_{p}-b_{p} a_{p-n}\right)\right)+\frac{1}{\Gamma} n^{2} b_{n} \\
-b_{n}\left(\frac{\partial \ln (D(\sigma) / D(0))}{\partial \sigma}\right)-\frac{T}{\epsilon} \frac{D(0)}{D(\sigma)} \sqrt{\frac{n}{2}}\left(a_{n}+b_{n}\right)
\end{array}\right.
$$

These equations are solved numerically using a spatial finite difference method, with the boundary condition $q_{+}(0, \theta)$, which is a time periodic function. At first order, the classical Euler method is used, and correction is performed using the Adams Moulton second-order method.

Although the simulation method has been verified for small propagation distances in Menguy and Gilbert (2000), it has to be tested for distances greater than the shock formation distance $\sigma_{c}$, in order to be used in brass instrument simulations. Tests have therefore been carried out for weakly dissipative fluids without any viscothermal wall effects $(T / \varepsilon=0)$ for which weakly nonlinear propagation can be described by the Burgers equation. One of them, defined by a Gold'berg number $\Gamma$ equal to $100(\Gamma>>1)$, is discussed now. 
The harmonic components, $P_{n}=\sqrt{a_{n}^{2}+b_{n}^{2}}$, as a function of $\sigma / \sigma_{c}$ varying from 0 to 10 for a wave generated by a mono frequency source are displayed in Figure 1. The behavior of the harmonic components simulated from equation 4 is close to the so-called Fay-Blackstock solutions available for $\sigma>3$. These analytic Fourier coefficients $Q_{n}$ are written as follows:

$$
q_{+}(\sigma, \theta)=\sum_{n=1}^{\infty} \sin (n \theta) Q_{n}(\sigma)=\frac{2}{\Gamma} \sum_{n=1}^{\infty} \frac{\sin (n \theta)}{\sinh [n(1+\sigma) / \Gamma]}
$$

Moreover simulations of a plane wave propagating in a uniform duct, defined by $T / \varepsilon=$ 10, are carried out from equation 4. The harmonic components as a function of $\sigma$ varying from 0 to 10 for a wave generated by a mono frequency source are displayed in Figure 1. The wave deformation along the propagation, from sine wave to a decreasing amplitude sawtooth wave, is obtained. The waveforms are typical of those reported in literature for high intensity sound in ducts (Hamilton and Blackstock, 1998): the boundary-layer losses are predominant, their dispersion effects are visible on the waveforms, and the Fay-Blackstock solutions are not a good approximation anymore. The spectral enrichment can be globally estimated from the following dimensionless parameter, called the spectral centroid $S C$ :

$$
S C=\frac{\sum_{n} n Q_{n}}{\sum_{n} Q_{n}}
$$

Figure 2 shows the rapidly increasing and then slowly decreasing evolution of $S C$, respectively before one and after three shock formation distance values corresponding to the results displayed in Figure 1, the shock formation distance $\sigma_{c}$ being still defined from the cylindrical tube.

Simulations of a plane wave propagating in a nonuniform duct, a cone, are also carried out from equation 1. The expanding bore of the cone implies a faster decay of the wave which reduces the nonlinear wave steepening; the spectral enrichment $S C$ increases more slowly than in a cylindrical tube having the same input radius (see Figure 2). 


\section{BRASSINESS SIMULATIONS}

\section{A. Numerical method summary}

The brassiness of the sound, in other words its spectral enrichment, generated by brass instruments at high dynamic level is mainly due to the essential nonlinearity of wave propagation in the pipe, resulting in wave steepening and generation of shock waves (Hirschberg et al, 1996). The spectral enrichment can be evaluated from the radiated pressure spectral centroid $S C$ estimation during a crescendo. In expanding bores, a faster decay of the wave amplitude reduces the nonlinear steepening effect: this provides a hypothesis to explain the fact that "conical" instruments are not as brassy as "cylindrical" instruments. More precisely, the brassiness of two brass instruments, or of two fingerings or positions of a given instrument, can be compared from their spectral centroid values for a given crescendo (Gilbert, 2006). The comparison can also be made by simulation, the input data being the internal geometry of the instrument, its bore, and typical acoustical pressures at the input end of the instrument.

The frequency model simulation method is based on the previous publications of Menguy and Gilbert (2000) and Gilbert et al (2005) dedicated to uniform ducts and clarinet-like instruments, and here adapted to non uniform ducts and brass instruments. The simulation can be summarized as follows: postulating the pressure spectrum $P_{\text {in }}$ at the input end, a radiated impedance boundary condition at the output end (radiation impedance formula from Caussé et al (1984), extended by the iterative impedance when $k . R$ is greater than 4.84 ), and the bore geometry of the instrument, the pressure and velocity acoustic field are first calculated everywhere inside the bore using the weakly nonlinear approximation. Indeed the simple waves propagating in each direction are supposed not to interact in the body of the fluid as in the linear approximation, and they are solutions of the nonlinear differential equations 1 and 2. The numerical solving of equation 4 and its twin has been presented in the previous section, the detail of the numerical method and a harmonic balance convergence method is detailed in Menguy and Gilbert (2000). The volume velocity spectrum $Q_{\text {out }}$ at 
the output end of the instrument is estimated by multiplying the output area by the output acoustic velocity spectrum. By using the low-frequency approximation for a monopole having a volume strength equal to $Q_{\text {out }}$, a radiated pressure spectrum $P_{\text {rad }}$ and its spectral centroid $S C_{r a d}$ are estimated at a distance $d$ from the open end of the pipe from:

$$
P_{\text {rad }}=\frac{\varrho}{4 \pi d} j \omega Q_{o u t}
$$

\section{B. Testing the model}

Simulations were done using the bore geometries of a bass trombone (Courtois model, 2000) corresponding to three slide positions in which F4 can be played: 1st, 4th and 6th positions (see Figure 3). From the 1st to the 4th (6th), there is an added cylindrical tube of $2 \times 0.27 \mathrm{~m}(2 \times 0.46 \mathrm{~m})$. Pressures spectra $P_{\text {in }}$ at the input end have been measured to provide input data for simulations. They come from experimental data collected in the side of the mouthpiece backbore during playing performance of a F4 using the bass trombone. One corresponding to loud playing having a $S C_{i n}$ value equal to 1.6 is displayed in Figure 4. The simulated radiated pressure is displayed too.

Several tests were performed to determine the reliability of the simulation method. Two kinds of crescendo have been chosen for simulations. One is created by the input pressure $P_{\text {in }}$ being a sine wave (spectral centroid $S C_{\text {in }}$ constant equal to 1), and having an increasing $R M S$ value from 500 to $3000 \mathrm{~Pa}$. The second is created by the input pressure $P_{\text {in }}$ increasing with a $R M S$ value from 500 to $3000 \mathrm{~Pa}$, its spectral centroid varying linearly from 1.22 to 1.62 (see Figure 5). The fundamental frequency is $350 \mathrm{~Hz}$ in both cases and the wave forms are defined by their first ten harmonics. The two simulated crescendos have been carried out using the three bore profiles displayed in Figure 3. The resulting values of the spectral centroid $S C_{\text {rad }}$ of the radiated sound are displayed in Figure 5. All the simulated spectral centroid values are increasing with the $R M S$ pressure $P_{i n}$ at the entrance of the instruments, showing the spectral enrichment of the radiated sound. As expected the spectral enrichment is greater and greater from the 1st to the 6th slide position because of the increasing length of 
the cylindrical part of the instrument. These predictions are qualitatively comparable with experimental results shown in Gilbert (2006). Note that the values of $S C_{\text {rad }}$ are depending on the input data $P_{i n}$ : results obtained using a variable $S C_{i n}$ are greater than the one obtained from $S C_{i n}$ constant equal to one.

\section{CONCLUDING REMARKS}

A frequency domain simulation tool has been developed to predict the brassiness behavior of brass instruments. It is based on the generalized Burger's equations 1 and 2 applied to weakly nonlinear acoustic propagation in non uniform ducts. First, numerical simulations of traveling waves in uniform ducts have been carried out over large distances to check their reliability far from the shock formation distance. The results have been successfully compared with known theoretical predictions. Secondly, in the weakly nonlinear propagation approximation, waves in ducts of finite length propagate in both directions, independently as in the linear limit, except for coupling at the ends. Then the internal sound field can be described by the superposition of two simple waves propagating in opposite directions which do not interact in the body of the fluid. This is the major hypothesis of the simulation tool already described in Menguy and Gilbert (2000), applied to cylindrical tubes of short length. In the present paper it has been extended to non uniform ducts, having lengths greater than realistic shock formation distances, and has been used with a bass trombone bore. The spectral enrichment of the radiated sound during crescendos has been simulated, and the results are comparable with experimental results already presented in conference (Gilbert, 2006; Gilbert et al, 2007).

While the numerical tool presented in the present paper is promising, it must be borne in mind that many simulations have to be carried out and compared with experimental results, in order to establish the reliability of the tool as a predictor of the brassiness of brass instruments defined by their bore (Myers et al, 2007). 


\section{References}

R. Caussé, J. Kergomard and X. Lurton, Input impedance of brass instruments - Comparison between experiment and numerical models, J.Acoust.Soc.Am. 75, 241-254, 1984.

J. Gilbert, J.P. Dalmont, and T. Guimezanes, Nonlinear propagation in woodwinds, Proc. Forum Acusticum, Budapest, 2005.

J. Gilbert, Differences between cylindrical and conical brass instruments, the nonlinear propagation point of view from experiments and simulations, Joint Meeting between ASA and ASJ, Hawaii, 2006.

J. Gilbert, D.M. Campbell, A. Myers, and R.W. Pyle, Difference between brass instruments arising from variations in brassiness due to non-linear propagation, Proceedings of International Symposium of Musical Acoustics, Barcelona, 2007.

M.F. Hamilton and D.T. Blackstock (eds), Nonlinear Acousics, Academic Press, 1998.

A. Hirschberg, J. Gilbert, R. Msallam and A.P.J. Wijnands, Shock waves in trombones, J.Acoust.Soc.Am. 99, 1754-1758, 1996.

L. Menguy, and J. Gilbert, Weakly non-linear gas oscillations in air-filled tubes ; solutions and experiments, Acustica 86, 798-810, 2000.

R. Msallam, S. Dequidt, R. Caussé, and S. Tassart, Physical model of the trombone including nonlinear effects, application to the sound synthesis of loud tones, Acustica 86, 725-736, 2000.

A. Myers,J. Gilbert, R.W. Pyle, and D.M. Campbell, Non-linear propagation characteristics in the evolution of brass musical instrument design, Proc. International Congress on Acoustics, Madrid, 2007.

M.W. Thompson, and W.J. Strong, Inclusion of wave steepening in a frequency-domain model of trombone sound production, J. Acoust. Soc. Am. 110, 556-562, 2001.

C. Vergez, and X. Rodet, New algorithm for Nonlinear Propagation of a sound Wave, Application to a Physical Model of a Trumpet, Journal of Signal Processing 4, 2000. 


\section{List of Figures}

FIG. 1 First three Fourier coefficients $Q_{n}$ (harmonics 1 to 3 ) versus the dimensionless propagation distance $\sigma / \sigma_{c}$ for a weakly dissipative fluid $(\Gamma=100)$ in uniform duct, according to two cases : (a) $T / \varepsilon=0$, and (b) $T / \varepsilon=10$. The fluid is excited at $\sigma=0$ by a mono frequency source. Two calculations: results simulated from equation 1 (solid lines) and Fay-Blackstock approximation, equation 5 (dashed lines). . . . . . . . . . . . . . .

FIG. 2 Spectral centroid $S C$ versus the dimensionless propagation distance $\sigma / \sigma_{c}$ for a cylindrical tube, and a cone having the same input radius as the cylindrical

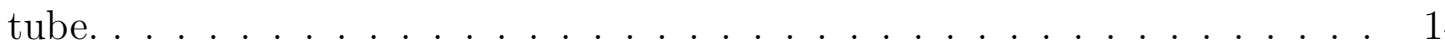

FIG. 3 The bore profiles corresponding to the three slide position (1st), (4th) and (6th), of a bass trombone versus the geometric position from the input end of the instrument without its mouthpiece.

FIG. 4 Dimensionless pressure spectra $P_{\text {in }}$ (circle) at the input end, and $P_{\text {rad }}$ (triangle) radiated, corresponding to a loudly played $F 4$ at the 1 st position.

FIG. 5 Spectrum centroid $S C_{\text {rad }}$ versus $R M S$ pressure of $P_{\text {in }}$ having an increasing value of $S C_{\text {in }}$ from 1.22 to 1.62 , simulations corresponding to the 1st (diamond), 4th (asterisk) and 6th (circle) positions. . . . . . . . . . . . 


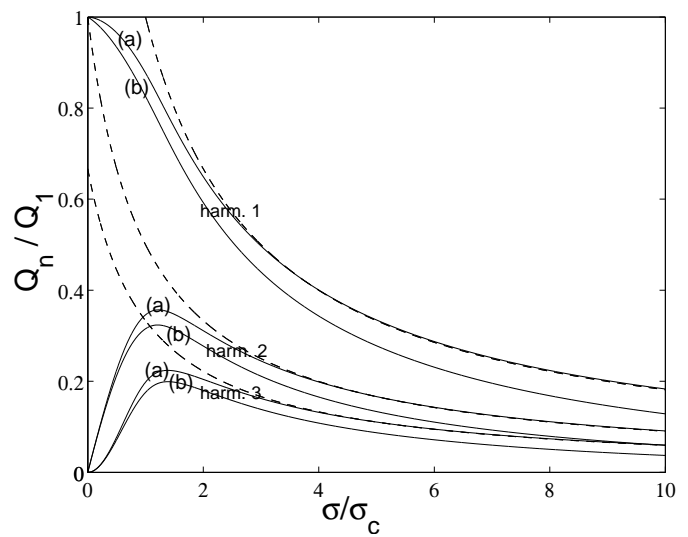

FIG. 1. 


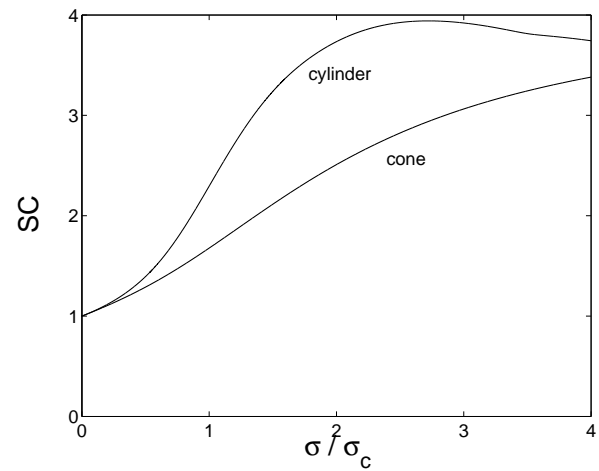

FIG. 2. 


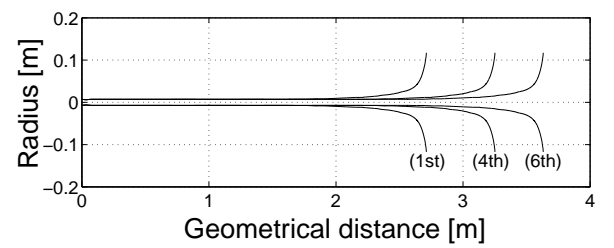

FIG. 3. 


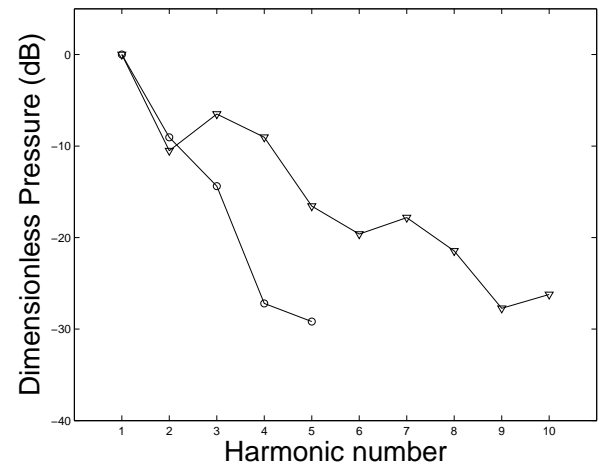

FIG. 4. 


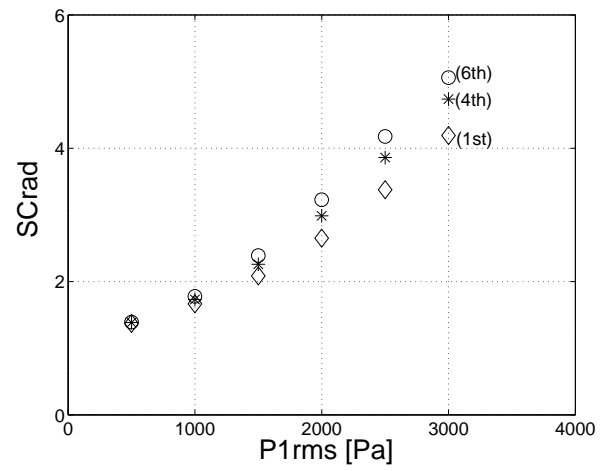

FIG. 5. 